\title{
Perfect retention of an instrumental response'
}

\author{
David Ehrenfreund and Joseph Allen \\ SOUTHERN ILLINOIS UNIVERSITY
}

\begin{abstract}
Abstraet
Retention tests for the running response after 27 days revealed a typical decrement for terminal speeds. Running speeds, however, showed no decrement. The difference between these data and previous data showing a decrement are analyzed in terms of the drive maintenance schedule in relation to the role of the $r_{g^{-}} g$ mechanism.
\end{abstract}

\section{Problem}

Studies by Gleitman \& Steinman (1963) purport to demonstrate forgetting of an instrumental running response in rats in the absence of any explicit interference either before or after the original training. Since Ss were not removed from their cages during the retention interval there was no opportunity for either extinction of the runway response or for the development of interfering responses. Furthermore, it was argued since the hunger drive (defined as a $22 \mathrm{hr}$. deprivation interval) was maintained throughout the retention interval the response decrement could not be ascribed to a change in Drive.

Although maintaining the hunger drive during the retention interval is obviously an important control this does not guarantee that the total motivational level has been maintained. According to contemporary behavior theory (Spence, 1956) total motivational level also depends upon the value of incentive motivation (K) a quantitative molar variable which reflects the strength of the fractional anticipatory response $\left(r_{g}\right)$. Any weakening of the $r_{g}-s_{g}$ mechanism related to the instrumental response of running would result in a deficit in performance as revealed by slower speed scores. A detailed S-R analysis of similarities between acquisition and retention strongly suggests that under the usual hours of deprivation technique of maintaining drive the anticipatory running response suffers extinction during the retention interval. During acquisition a variety of auditory, olfactory, and visual cues which regularly precede data collection would come to elicit the $\mathrm{r}_{\mathrm{g}}{ }^{-\mathrm{s}_{\mathrm{g}}}$ mechanism related to running in the alley. But these cues are highly similar, often identical to the cues involved in approaching the cage to feed the animal as part of the drive maintenance schedule. Through generalization the latter cues would also elicit the $r_{g^{-}} s_{g}$ mechanism. Whatever effect such an S-R association has during acquisition, it is obvious that as regards the anticipatory goal response to the alley, the retention interval represents a classical extinction procedure. Thus, the similarity between stimuli associated with a daily feeding and the stimuli associated with data collection may have behaviorial consequences which significantly affect the data in retention studies with rats.
In the present study, the hunger drive is maintained in such a way as to preclude the possibility of either interference or the weakening of relevant $\mathrm{r}_{\mathrm{g}}-\mathrm{s}_{\mathrm{g}}$ mechanisms during the retention interval. Under our procedure (Ehrenfreund, 1960) Ss are maintained at a particular body weight continuously and automatically while living in a weight-control apparatus which senses a slight weight loss (2 gm), and delivers small amounts of food $(1 / 3 \mathrm{gm})$ until the loss is recovered. Thus the periodicity of food presentation related to the drive maintenance schedule is entirely unrelated to the periodicity of running in the alley; food presentations are not associated with cues arising from the presence and activity of the experimenter or animal caretaker; $S$ is never removed from its cage except to be run in the alley; drive is maintained continuously rather than cyclically; S's percentage weight, which defines drive level, remains constant within a $2 \mathrm{gm}$ limit.

\section{Method}

Ss were 14 Sprague Dawley male rats, five months old at the start of the experiment. All Ss had been reduced to $80 \%$ of free weight at age 100 days and maintained at this level in individual weight-control devices until the end of the present study. These devices, similar to those previously described (Ehrenfreund, 1960), assures that S is always within $1 \%$ of a specified weight.

A new food delivery mechanism using powdered food is now used. Thus enough food and water can be supplied so that the device does not have to be attended to for 2 or 3 days, although we never let more than a day pass without checking. Next to the room containing the weight-control device is a smaller room containing the runway which is a copy of one previously described (Ehrenfreund \& Badia, 1962). Essentially it is $5 \mathrm{ft}$ long, $21 / 2$ in wide, and 4 in high. It is constructed of cloudy plastic so that the interior presents a continuous, homogeneous, and diffusely illuminated environment to the S. Filtered light beams from above the maze were directed to photocells below the maze. The response measures were (a) running speed which involved the middle $2 \mathrm{ft}$ section of the maze and (b) terminal speed which involved the last 18 in of the maze.

All Ss received 5 massed trials a day for 17 days, by which time all Ss had clearly reached asymptote. For the next 27 days each $S$ continued to live in its own weightcontrol device. For half the Ss the device was set to maintain them at $90 \%$ of free weight. During the 27 day interval the food hopper and water bottle were checked and refilled as necessary. Since both were outside the cage, neither the cage nor $S$ were disturbed. Each $S$ then received 5 trials a day for 3 days exactly as in acquisition, except for the reduced drive for half of the Ss. 


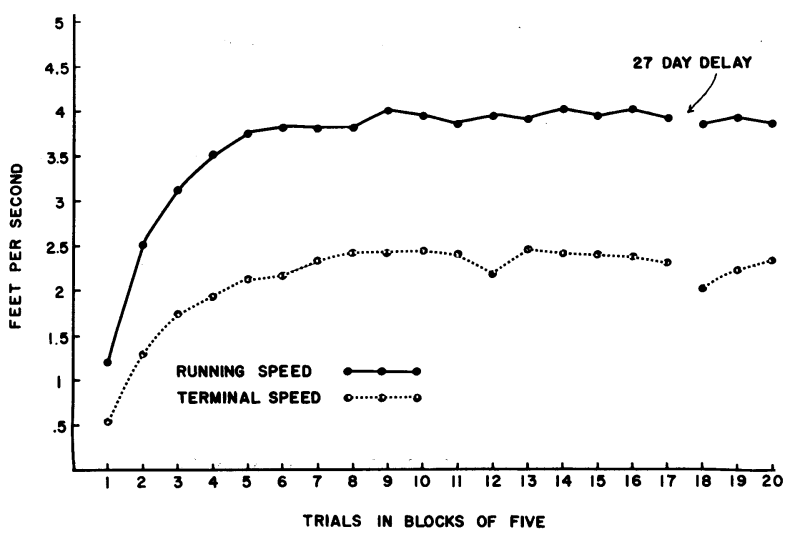

Fig. 1. Acquisition and retention for both running and terminal speeds. Each point on the curve is the mean of the individual S's median score.

\section{Results}

For the purpose of the present study we need only compare the last trial of acquisition with the first trial of retention, 27 days later. Other analyses give essentially the same result and this comparison should be the most sensitive. Although the difference in drive produced results in the expected direction it failed of significance $(p<.10)$ and therefore both drive groups were combined. There was also no drive-interval interaction.

Running and terminal speeds produced quite different retention results. The terminal speeds suffered a decrement over the 27 day retention interval. The difference between the last acquisition and first retention trial was significant beyond the .005 level. This loss, indicative of forgetting is consistent with previous studies. Turning now to the data for running speeds we find a very different relationship. Between the last trial of acquisition and first trial of retention the $\mathrm{F}$ was 1.25 which for $1 / 13 \mathrm{df}$ is probable between the .10 and .25 levels. Several other statistical analyses were made and all led to the same conclusion namely for running speed there was no loss that was not attributable to chance. The actual mean difference over the 27 day retention interval was $.02 \mathrm{sec}$. whereas on the average it took .53 sec. for a $\mathrm{S}$ to run the middle $2 \mathrm{ft}$ section.

\section{Discussion}

White rats can and do acquire both excitatory and inhibitory tendencies in the living cage that affect subsequent performance. This is dramatically revealed in a comparison of the study by Birch, Bernstein, \& Clark (1958) with a modified replication by Brown \& Belloni (1963). In both studies Ss were maintained on a $22 \mathrm{hr}$. deprivation interval for an extended period of time (35 days) prior to being tested for running speed, under varying hours of deprivation. Whereas Birch et al. (1958) reported a peaking at or just beyond the $22 \mathrm{hr}$. value with a sharp drop in performance for tests at shorter or longer deprivation intervals, Brown \& Belloni (1963) reported a flat gradient with no drive effect. Tests of approach response to the food trough as a function of deprivation revealed a similar bidirectional gradient for the Birch et al. study but again a flat gradient for the Brown et al. study. In the earlier study the food trough remained in the individual cage all the time whereas in the modified replication the food trough was in the cage only during the daily $2 \mathrm{hr}$. feeding. Brown \& Belloni discussed the importance of this change as it affected the trough depressions, pointing to the possibility of extinction of the approach response to the trough at times other than $22 \mathrm{hr}$. and therefore the development of a discrimination. In their own study, since the food trough was present only during feeding, approach responses to the trough could not be made at other times; therefore, could not be extinguished, thus precluding the development of temporal discrimination. In terms of the analysis made earlier in the present paper we would suggest that the difference between the two studies in respect to running speeds was also due to the consequences of leaving the food trough in the cage all the time vs. only during feeding.

Independently of whether our results are due to the preclusion of $\mathrm{r}_{\mathrm{g}}-\mathrm{s}_{\mathrm{g}}$ weakening, it is now possible in studies employing instrumental running, to systematically investigate laws of forgetting. The "extinction of $\mathrm{r}_{\mathrm{g}}$ " hypothesis could be investigated by a controlled variation of cues related to the elicitation of $r_{g}$. In another sense the data of the present study makes it difficult to continue to ignore the relationship between the studies of the temporal distribution of trials and retention. In fact, why should these two bodies of knowledge not be considered as being on the same continuum? References

BIRCH, D., BURNSTEIN, E., \& CLARK, R. Response strength as a function of hours of food deprivation under a controlled maintenance schedule. J. comp. physiol. Psychol., 1958, 51, 350-354.

BROWN, J., \& BELLONI, M. Performance as a function of deprivation time following periodic feeding in an isolated environment. J. comp. physiol. Psychol., 1963, 56, 105-110.

EHRENFREUND, D. The motivational effect of a continuous weight loss schedule. Psychol. Rep., 1960, 6, 339-345.

EHRENFREUND, D., \& BADIA, P. Response strength as a function of drive. level and pre- and postshift incentive magnitude. J. exp. Psychol., 1962, 63, 468-471.

GLEITMAN, H., \& STEINMAN, F. The retention of runway performance as a function of proactive interference. J.comp. physiol. Psychol., 1963, 56, 834-838.

SPENCE, K. W., Behavior theory and conditioning. New Haven: Yale Univer. Press, 1956.

\section{Note}

1. Conduct of this research and preparation of this paper were supported by a grant from the USPHS Research Grant MH 07157 from the Institute of Mental Health, United States Public Health Service. 